\title{
Contingency Management for the Treatment of Antipsychotic-Induced Weight Gain: A Randomized Controlled Pilot Study
}

\author{
Joseph C. Ratliff Laura B. Palmese K.Melek Tonizzo Lydia Chwastiak \\ Cenk Tek \\ Department of Psychiatry, Yale University School of Medicine, New Haven, CT, USA
}

\section{Key Words}

Obesity management $\cdot$ Schizophrenia $\cdot$ Weight gain

\begin{abstract}
Objective: Weight gain is common for individuals with serious mental illness (SMI) receiving antipsychotic drug therapy. Contingency management (CM) is a behavioral intervention that rewards positive performance and has demonstrated effectiveness in reducing drug use in SMI populations. This study evaluated the feasibility of using $\mathrm{CM}$ to promote weight loss in individuals with SMI over 8 weeks. Method: 30 individuals (BMI $\geq 28 \mathrm{~kg} / \mathrm{m}^{2}$ ) were randomized to one of three conditions: i) The combination of a standardized lifestyle modification (LM) program for individuals with SMI and payment for group attendance ( $\left.\mathrm{CM}_{\text {attendance }}\right)$, ii) The combination of $L M$ and payment for weight loss $\left(\mathrm{CM}_{\text {weight }}\right)$, and iii) waitlist control (CON). After the waitlist period, those participants joined a LM group and received payment for behavioral change ( $\left.\mathrm{CM}_{\text {behavior }}\right)$. Results: Subjects in the $\mathrm{CM}_{\text {attendance }}$ and in the $\mathrm{CM}_{\text {weight }}$ group lost a mean of $1.16 \mathrm{~kg}$ and $1.23 \mathrm{~kg}$, respectively, while subjects in the CON gained a mean of 0.68

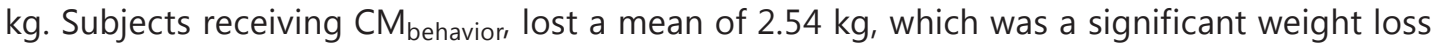
compared to the control period. Conclusion: LM supplemented with CM may facilitate weight loss in patients taking antipsychotic medications; financial reimbursement for behavioral change may be particularly effective in this population.

Copyright (c) 2012 S. Karger GmbH, Freiburg
\end{abstract}

\section{Introduction}

Individuals with severe mental illness (SMI) have a life expectancy that is 2 decades shorter than that of the general population [1]. SMI is associated with increased prevalence rates of obesity [2], type 2 diabetes [3], and cardiovascular disease [4]. Poor nutritional 
choices, sedentary lifestyle, and antipsychotic medications (particularly some 2nd-generation antipsychotic medications) contribute to the increased obesity rates observed in SMI populations [5, 6]. Lifestyle modification (LM) interventions can be utilized as a first-line treatment for obesity and produce approximately $0.5 \mathrm{~kg} /$ week of weight loss in the general population [7]. LM and cognitive behavioral therapy have been shown to reduce weight or prevent weight gain in SMI populations [8-11]; a review of randomized controlled studies reported that these techniques average approximately $0.2 \mathrm{~kg} /$ week of weight loss over 15 weeks in this population [12]. There is a critical need to develop strategies to augment the effectiveness of LM interventions in SMI populations.

Contingency management (CM) is based on the behavioral principle that reinforcement of a targeted behavior will increase its frequency. CM has been used extensively to facilitate behavior change in individuals with substance abuse disorders [13]. CM is applied to situations in which an outcome is easily detected (e.g., abstinence from substances), and individuals are rewarded, usually financially, for objective evidence of success [14, 15].

Applications of CM have included rewards for both attendance (adherence to treatment) and treatment outcomes (negative urine samples) [16, 17]. Nearly one third of all clinical appointments are missed [18], leading to lost opportunities for therapy and attrition from treatment. The effectiveness for CM for improving substance abuse outcomes has been demonstrated among individuals with SMI $[16,18]$. In one study, the provision of take-home doses of methadone significantly improved attendance at therapy sessions [19]. In a different study of CM, monetary incentives increased the number of negative urine samples in a sample of SMI patients that abused marijuana [16].

As CM has been used to increase gym attendance [20] and to reinforce healthy eating habits [21] and financial incentives have been shown to promote weight loss in obese individuals [22-24], the combination of LM with CM may be particularly effective in promoting weight loss. In a previous study, we demonstrated the effectiveness of CM for behavioral change (in the form of food reimbursement) in promoting weight loss among overweight and obese persons with SMI [12]. This intervention allowed participants to shop for healthy foods according to their own tastes, which made individuals more receptive to dietary change by incorporating their own preferences while also providing a counseling opportunity to guide them on making better food choices. Food reimbursement resulted in significant weight loss compared to that of the control group, and participants continued to lose weight 6 months after the intervention. LM in conjunction with financial incentives as a reward contingent on weight loss or other behavior change represents a novel treatment for antipsychotic-induced weight gain $[25,26]$.

The objective of this study was to determine the feasibility and efficacy of an 8-week LM program utilizing CM for the treatment of antipsychotic-induced weight gain in individuals with SMI. We aimed to determine whether there would be any difference in weight loss across groups which were rewarded either for attendance, weight loss, or behavioral change. We hypothesized that rewarding for behavioral change would be the most effective based on our previous experience with food reimbursement.

\section{Subjects and Methods}

Subjects

30 individuals (BMI $\geq 28 \mathrm{~kg} / \mathrm{m}^{2}$ ) aged 18 to 70 years with SMI who were on a stable dose of antipsychotics for at least 1 month and self-reported weight gain $\geq 5 \%$ over the past 5 years were recruited via advertisement or referral. Individuals who were pregnant, had a history of dementia or of mental retardation, or were not fluent in English were excluded from the study. 
This study was conducted from October 2010 through March 2011 at the Connecticut Mental Health Center, in New Haven, CT. The protocol was approved by the Institutional Review Board of Yale University. After complete description of the study to the participants, written informed consent was obtained.

\section{Lifestyle Modification}

All subjects received an 8-week, randomized, group-based LM weight loss intervention coupled with $\mathrm{CM}$ in the form of financial reimbursement. Initially, participants were randomly assigned by a blinded research assistant using randomization software to one of three groups; i) contingency for weight $\left(\mathrm{CM}_{\text {weight }}\right)$, ii) contingency for attendance $\left(\mathrm{CM}_{\text {attendance }}\right)$, or iii) waitlist control (CON). After 8 weeks, participants from $\mathrm{CON}$ joined a group that had a contingency for behavioral change $\left(\mathrm{CM}_{\text {behavior }}\right)$ in the form of reimbursement for healthy food purchases. This was chosen to follow CON because we have demonstrated the effectiveness of rewarding behavioral change with food reimbursement in a SMI population previously [12].

The LM program was the Simplified Intervention to Modify Physical Activity, Lifestyle and, Eating Behavior (SIMPLE) program, a standardized lifestyle program based on social cognitive theory. SIMPLE was specifically designed to be used with SMI populations, assumed no prior nutritional knowledge, and all concepts were presented in basic terms with cultural sensitivity [12]. The format consisted of weekly, hour-long group sessions with 8-10 individuals. Topics focused broadly on nutrition and physical activity and included changing attitudes to support weight loss and implementing social support to aid in weight loss. The main focus of the nutritional component of SIMPLE was to help participants choose healthy options from each food group while reducing total calorie consumption. The physical activity component focused on helping participants identify ways to increase daily physical activity (e.g., walking more and choosing stairs rather than elevators).

\section{Contingencies for Weight and Attendance}

All participants were paid USD 20.00 for each of the baseline and week 8 assessments. The total maximum dollar amount (USD 140.00) that individuals could potentially earn for contingencies was the same across the three different types of CM groups; however, the interventions differed with respect to the way money was earned. Individuals $(n=10)$ in the $\mathrm{CM}_{\text {attendance }}$ group received USD 17.50 for each of the 8 weight loss groups they attended. Individuals $(n=10)$ in the $\mathrm{CM}_{\text {weight }}$ group received monetary reward for weight loss. Starting in the 2 nd week of group, if a group member lost at least $1 \mathrm{lb}$ since the previous week, a USD 5.00 reward was given. At week 3, if the group member lost at least another pound, the payment was USD 10.00. Payments continued to escalate in USD 5.00 increments for each week that the group member lost at least $1 \mathrm{lb}$. If a group member was unable to lose at least $1 \mathrm{lb}$, no payment was disbursed, and payments would reset back to the USD 5.00 level.

\section{Waitlist Control and Contingency for Behavior}

Individuals $(\mathrm{n}=10)$ randomized to the CON group received no specific dietary or physical activity intervention for 8 weeks and were instructed to continue with their habitual patterns of eating and activity. After the 8-week period was over, subjects entered a LM group with a contingency for behavior $\left(\mathrm{CM}_{\text {behavior }}\right)$. During the $\mathrm{CM}_{\text {behavior }}$ group intervention, participants received financial reimbursement for healthy foods that were purchased from a shopping list that was handed out the first week of group. Starting in the 2nd week, group members brought their grocery receipts to group and were reimbursed up to USD 20.00 a week for healthy items purchased. All intervention groups received the healthy food list during the first session, but only the $\mathrm{CM}_{\text {behavior }}$ group was reimbursed for healthy food purchases.

\section{Outcome Measures and Other Assessments}

Weight was measured weekly before group sessions. All other ratings were performed at baseline and after 8 weeks. The primary outcome measure was change in weight after 8 weeks.

\section{Laboratory and Anthropometric Measurements}

Weight was measured in light clothing, without shoes, to the nearest $0.1 \mathrm{~kg}$, and height was measured to the nearest $0.1 \mathrm{~cm}$ on a calibrated, portable scale (Pelstar LLC, Alsip, IL, USA). BMI was calculated as $\mathrm{kg} / \mathrm{m}^{2}$. Waist circumference was the average of two measurements at the midpoint between the iliac crest and the lowest rib to the nearest $0.1 \mathrm{~cm}$. Blood pressure was measured using a calibrated wrist monitor (Oregon Scientific, Portland, OR, USA) after participants sat quietly for $5 \mathrm{~min}$. 
Plasma glucose levels (YSI, Yellow Springs, OH, USA), plasma insulin concentrations (Diagnostic, Webster, TX, USA), glycosylated hemoglobin (HbA1c) (Miles, Inc., Elkhart, IN, USA), and lipid profile (Sigma, St. Louis, MO, USA) were measured using standard enzymatic procedures.

Questionnaires

A 24-hour diet recall [27] was used to gather information about food and beverage consumption. Recalls were semi-structured interviews conducted with trained research staff. The Godin Leisure Time Exercise Questionnaire (GLTEQ) [28] was used to assess self-reported frequency of mild, moderate, and vigorous exercise.

\section{Statistical Analysis}

A preliminary sample size calculation determined that 8 members in each group would provide $80 \%$ power to detect a significant weight loss difference based on subjects in the $\mathrm{CM}_{\text {behavior }}$ group losing $1 \mathrm{lb} /$ week, subjects in the $\mathrm{CM}_{\text {attendance }}$ and $\mathrm{CM}_{\text {weight }}$ groups losing $0.5 \mathrm{lb} /$ week, and no weight change for CON. Subjects who completed baseline and 8-week assessments were included in the outcome analyses. Analysis of Covariance (ANCOVA) was conducted with $\mathrm{CM}_{\text {attendance, }} \mathrm{CM}_{\text {weight }}$, and CON groups for the 8-week intervention. A repeated measures analysis of variance (ANOVA) was used to assess results for the CON group that became the $\mathrm{CM}_{\text {behavior }}$ group. Finally, the three CM groups were compared using ANCOVA with chlorpromazine equivalent, sessions attended, and contingencies earned as covariates. Bivariate correlations were calculated using Spearman's correlation coefficient to assess the relationship between session attendance, body weight, physical activity, eating behaviors, and quality of life. All analyses were performed using SPSS, version 17.0 for Windows (SPSS Inc., Chicago, IL, USA).

\section{Results}

\section{Participant Characteristics}

26 individuals $\left(\mathrm{CM}_{\text {attendance }}=9, \mathrm{CM}_{\text {weight }}=9, \mathrm{CON}=8\right)$ completed the first 8 weeks of the study, and 9 individuals from the CON group completed $\mathrm{CM}_{\text {behavior }}$ after the waitlist period. The flow of study participants is presented in figure 1 . Subjects primarily had schizophrenia or schizoaffective disorder diagnoses (60\%). Three individuals $\left(\mathrm{CM}_{\text {attendance, }} \mathrm{CM}_{\text {weight }}, \mathrm{CON}\right)$ dropped out of the study after randomization. One subject in the CON group was excluded from CON analyses because a weight was not available after the waitlist period; however, this individual was allowed to join $\mathrm{CM}_{\text {behavior }}$ as scheduled. Baseline antipsychotic medication dose was statistically different between groups based on calculated chlorpromazine equivalencies [29] and was therefore controlled for during subsequent analyses. The average length of illness was $23 \pm 13$ years. There were no differences in age, gender, ethnicity, length of illness, or psychiatric diagnosis between any of the groups, and there were no differences between dropouts and those that completed the study. Demographic characteristics are presented in table 1.

\section{Anthropometric Values}

There were no differences in weight or BMI between groups at baseline. After 8 weeks, subjects in both the $\mathrm{CM}_{\text {attendance }}(-1.16 \pm 2.34 \mathrm{~kg})$ and the $\mathrm{CM}_{\text {weight }}(-1.23 \pm 2.01 \mathrm{~kg})$ groups lost weight, while CON subjects, on average, gained weight $(0.68 \pm 2.66 \mathrm{~kg})$, although these differences did not reach statistical significance. CM also reduced waist circumference, while this measure increased among CON subjects, but these differences were also not statistically significant. A repeated measures ANOVA showed that $\mathrm{CM}_{\text {behavior }}$ resulted in a significant weight loss $(-2.54 \pm 2.49 \mathrm{~kg})$ compared to the 8-week waitlist control period $(F(1,8)=9.63, p=.02$, table 2$)$. Similar analyses also showed that BMI was significantly improved for subjects after the $\mathrm{CM}_{\text {behavior }}$ intervention $\left(-0.92 \pm 0.92 \mathrm{~kg} / \mathrm{m}^{2}\right)$ compared with the control period $\left(0.34 \pm 0.87 \mathrm{~kg} / \mathrm{m}^{2} ; \mathrm{F}(1,8)=11.08, \mathrm{p}=0.01\right)$. There 
Fig. 1. CONSORT flow diagram.

Ratliff et al.: Contingency Management for the Treatment of Antipsychotic-Induced Weight Gain: A Randomized Controlled Pilot Study

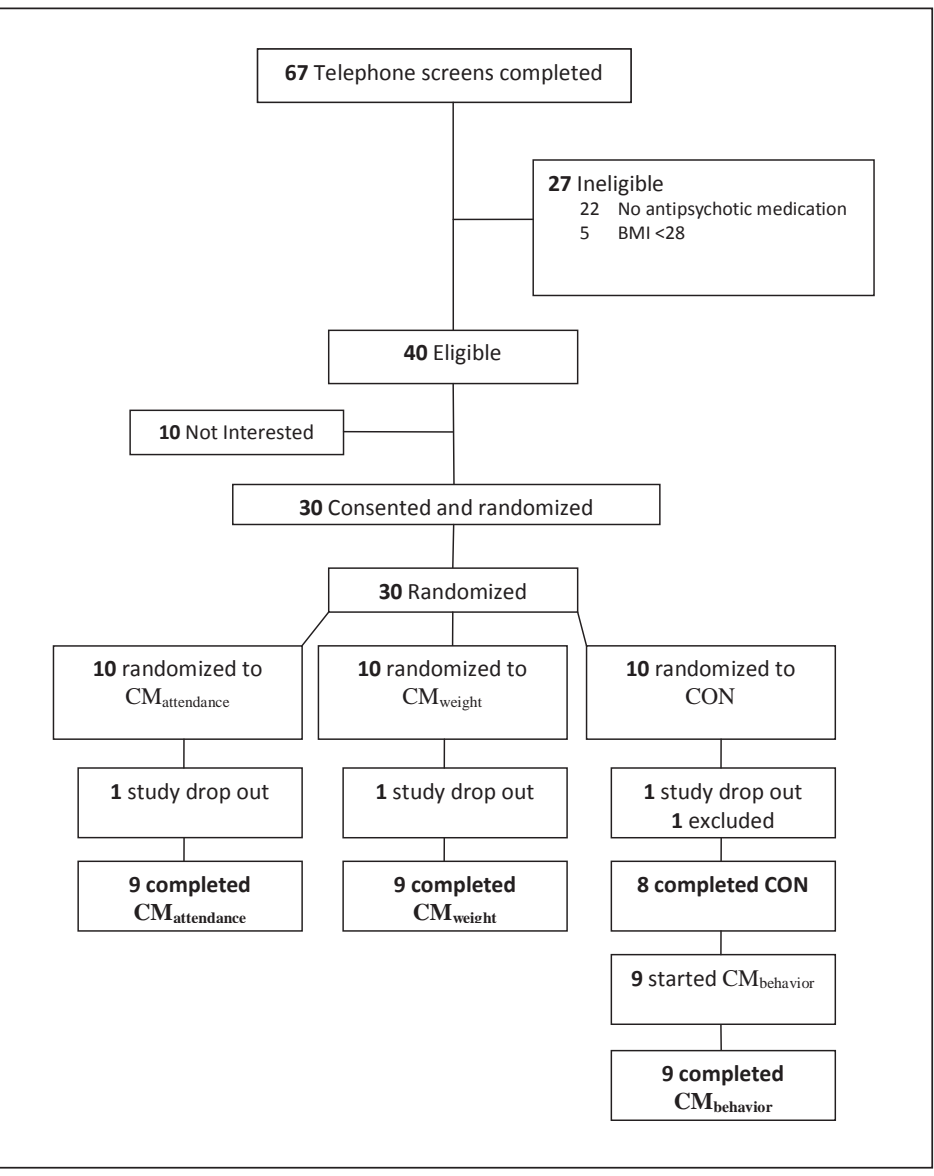

Table 1. Demographic information ${ }^{\mathrm{a}}$

\begin{tabular}{llllll}
\hline Variable & $\mathrm{CM}_{\text {weight }}(\mathrm{n}=10)$ & $\mathrm{CON}(\mathrm{n}=10)$ & $\mathrm{CM}_{\text {attendance }}(\mathrm{n}=10) \mathrm{F}$ or $\chi^{2}$ & P value \\
\hline Female, $\%$ & 60 & 70 & 70 & 1.82 & 0.178 \\
Age, years & $50.1 \pm 10.6$ & $47.3 \pm 8.0$ & $49.0 \pm 8.1$ & 0.19 & 0.829 \\
$\mathrm{C} / \mathrm{AA} / \mathrm{H}, \mathrm{n}$ & $6 / 3 / 1$ & $3 / 4 / 3$ & $6 / 4 / 0$ & 2.67 & 0.264 \\
$\mathrm{BMI}, \mathrm{kg} / \mathrm{m}^{2}$ & $41.6 \pm 9.9$ & $38.1 \pm 5.7$ & $36.0 \pm 8.9$ & 1.02 & 0.376 \\
$\begin{array}{l}\text { Chlorpromazine } \\
\text { equivalent antipsy- }\end{array}$ & $481 \pm 414$ & $269 \pm 180$ & $833.33 \pm 617$ & 3.75 & 0.038 \\
chotic dosage, mg & & & & \\
\hline
\end{tabular}

$\mathrm{C}=$ Caucasian; $\mathrm{AA}=$ African American; $\mathrm{H}=$ Hispanic.

${ }^{a}$ Age, BMI, and antipsychotic dosage are reported as mean \pm SD.

were no differences in blood pressure between groups or over time. There were no significant differences between changes in outcome variables for the three CM groups after the intervention. 
Table 2. Changes in outcome variables between waitlist period and active intervention ${ }^{\mathrm{a}}$
Ratliff et al.: Contingency Management for the Treatment of Antipsychotic-Induced Weight Gain: A Randomized Controlled Pilot Study

\begin{tabular}{|c|c|c|c|c|}
\hline Variable & CON & $\mathrm{CM}_{\text {behavior }}$ & $\mathrm{F}^{\mathrm{b}}$ & $\mathrm{P}$ \\
\hline$\Delta$ Weight, kg & $0.68 \pm 2.66$ & $-2.54 \pm 2.49$ & 9.63 & 0.02 \\
\hline$\Delta \mathrm{BMI}, \mathrm{kg} / \mathrm{m}^{2}$ & $0.4 \pm 0.9$ & $-0.9 \pm 0.9$ & 11.08 & 0.01 \\
\hline$\Delta$ Waist, $\mathrm{cm}$ & $3.4 \pm 7.0$ & $-3.5 \pm 5.6$ & 0.93 & 0.39 \\
\hline$\Delta$ Glucose, mg/dl & $8.4 \pm 30.4$ & $-15.6 \pm 57.9$ & 0.95 & 0.76 \\
\hline$\Delta \mathrm{HbA} 1 \mathrm{c}, \%$ & $0.7 \pm 1.6$ & $-0.2 \pm 0.9$ & 0.80 & 0.41 \\
\hline$\Delta$ Insulin, uU/l & $-4.6 \pm 5.9$ & $4.3 \pm 6.7$ & 4.42 & 0.07 \\
\hline$\Delta \mathrm{TC}, \mathrm{mg} / \mathrm{dl}$ & $-4.3 \pm 20.8$ & $-0.6 \pm 24.9$ & 0.09 & 0.77 \\
\hline$\Delta$ Trig, mg/dl & $7.1 \pm 38.8$ & $0.0 \pm 31.4$ & 0.12 & 0.74 \\
\hline$\Delta \mathrm{HDL}, \mathrm{mg} / \mathrm{dl}$ & $-2.9 \pm 7.3$ & $4.3 \pm 4.4$ & $4 . .42$ & 0.06 \\
\hline$\Delta$ LDL. mg/dl & $-16.3 \pm 31.1$ & $3.7 \pm 24.8$ & 2.03 & 0.21 \\
\hline$\Delta$ Calories & $44 \pm 973$ & $-414 \pm 679$ & 0.54 & 0.50 \\
\hline$\Delta$ GLTEQ & $-5.5 \pm 35.0$ & $12.1 \pm 19.4$ & 0.41 & 0.55 \\
\hline \multicolumn{5}{|c|}{$\begin{array}{l}\text { HbA1c = Glycosylated hemoglobin; } \mathrm{TC}=\text { total cholesterol; Trig = } \\
\text { riglycerides } \\
\text { a Values are reported as means } \pm \mathrm{SD} . \\
{ }^{\mathrm{b}} \text { Repeated measures ANOVA. }\end{array}$} \\
\hline
\end{tabular}

\section{Laboratory Values}

There were no differences in laboratory values at any time point between any groups. CM tended to improve HDL cholesterol values, whereas this measurement worsened among CON subjects.

\section{Caloric Intake and Physical Activity}

There were no significant differences in caloric intake or physical activity between any

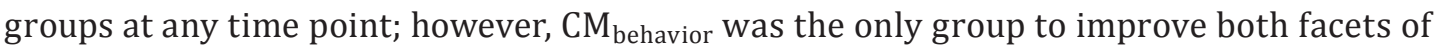
energy balance.

\section{Contingencies and Sessions Attended}

The percentage of sessions attended were $81 \%$ for $\mathrm{CM}_{\text {attendance, }} 78 \%$ for $\mathrm{CM}_{\text {behavior, }}$ and $60 \%$ for the $\mathrm{CM}_{\text {weight }}$ group. Contingencies earned for the $\mathrm{CM}_{\text {attendance }}$ (USD 112.78 / subject) and $\mathrm{CM}_{\text {behavior }}$ (USD 93.80 / subject) groups were significantly greater than those earned in the $\mathrm{CM}_{\text {weight }}$ group (USD 12.22 / subject) $(\mathrm{F}(2,27)=40, \mathrm{p} \leq 0.001)$. Percentage of sessions attended was positively correlated with baseline emotional health $(r=0.407, p \leq 0.05)$ and total GLTEQ score after treatment $(\mathrm{r}=0.452, \mathrm{p} \leq 0.05)$.

\section{Discussion}

Our study demonstrates the feasibility of combining CM with LM for weight loss among an overweight and obese SMI population. The study evaluated three different CM interventions (rewarding either attendance, weight loss, or the behavior of purchasing healthy foods). Mean weight was reduced in all of the intervention groups, regardless of the form of $\mathrm{CM}$ used. The magnitude of mean weight loss after 8 weeks (approximately $4 \mathrm{lb}$ ) was similar to results from other behavioral weight management studies on SMI populations and those from currently available pharmacological treatment studies using orlistat or phentermine [30]. 
In the present study, CM in the form of reimbursement for healthy food purchases resulted in the greatest weight loss, which is consistent with findings from a systematic review of studies using financial incentives for weight loss that reported a trend in favor of greater weight loss with reward for behavioral change rather than reward for weight loss outcome [31]. The increased cost of healthy foods is often cited as an impediment to appropriate food choices - especially for groups with low socioeconomic status [32, 33]. The combination of food provision and financial incentives may enhance weight loss because these strategies target both the antecedents and consequences of behavior, allowing participants to make better choices and learn from their past experiences.

In contrast, the $\mathrm{CM}$ interventions that rewarded either attendance or weight loss did not reduce weight more effectively when compared with the control group. Wing et al. [34] found no difference between the effects of financial incentives for weight loss and payment for group attendance in a group of overweight adults. It is possible that the method of payment for weight loss in this study limited its efficacy. Financial contracting, in which the participant deposits their own money, which is reimbursed contingent on weight loss, has been shown to substantially reduce weight in the general population [22]. Perhaps the threat of losing one's own money provides a greater incentive than receiving an external reward $[35,36]$, but this method would likely limit study volunteers for a population with limited socioeconomic means such as those with SMI. Another possibility is that the $1 \mathrm{lb} /$ week weight loss goal may have been too ambitious for this population. Previous behavioral studies have reported that weight loss over time is associated with increased session attendance [37]; however, the current study found no such relation. One possible explanation could be that individuals who were rewarded for attendance became passive participants in the LM process without positive reinforcement for changing eating behaviors or achieving weight loss.

We were unable to observe significant changes in metabolic indices after this intervention, likely due to the small sample size along with the abbreviated timeframe. Even modest weight loss can significantly improve glucose and lipid metabolism in SMI populations [38], making the improvement in HDL cholesterol levels observed with LM in the current study encouraging.

Session attendance was significantly correlated with baseline emotional health and frequency of physical activity at week 8. Developing strategies to improve emotional wellness may make participants more receptive to the behavioral changes necessary for weight loss. The increase of physical activity observed with higher session attendance was a positive outcome. Regular physical activity can decrease triglyceride and insulin concentrations for individuals with SMI [39]. While the sample size was too small to detect significant changes in metabolic parameters, the largest weight loss was observed in the only group that improved both their dietary and exercise patterns.

The primary limitations of this study were the small sample size, the brief duration, and the lack of long-term follow-up of the intervention which might have limited the ability to find significant differences in some outcome variables. The small sample size also did not allow for a differential analysis of effects for individual antipsychotics on weight change. Another limitation was the control group crossing-over to a CM group; it would have been less confounding to have four separate groups of individuals running simultaneously, but the time necessary to recruit another group would have increased study attrition. The use of a group receiving LM without incentives would have provided a direct comparison for the efficacy of CM in this population. There was selection bias in the recruitment of participants because these individuals were motivated to lose weight; so these results may not be indicative of the SMI population. Another limitation is the use of one 24-hour dietary recall. Some patients may underestimate or overestimate their actual intake or consume a different 
amount of food depending on whether it was a weekday or weekend. To minimize this variation, we provided measuring cups and food models to help patients visualize portion sizes, and dietary recalls were assessed on an equal number of weekdays and weekends. The use of self-reported physical activity frequency provides another limitation. Patients may have difficulty remembering duration and frequency of physical activity. Although objective measures like accelerometers may be more accurate, the GLTEQ provides clear examples to guide patients and is more suitable for a clinical setting. Another limitation was the lack of baseline cognitive testing. Perhaps limited cognitive functioning impaired some patients' ability to understand the LM information, or possibly there were differences in cognitive functioning between groups. Finally, the pilot was not designed to provide cost-effectiveness analyses. One criticism of CM that has reduced its implementation in community-based settings is cost [40]. Food reimbursement was associated with significant weight loss for less than USD 100.00 / patient over 8 weeks. Future research should try to determine the sustainability of $\mathrm{CM}$ and the lowest payment amount where benefits are still observed.

We found that an 8-week intervention that combined LM and CM for individuals with SMI reduced weight. Although rewarding participants for attendance, weight loss, or food purchasing behaviors were all associated with weight loss, food reimbursement (rewarding behavioral change) may be particularly effective in this population. CM may provide sufficient motivation to help SMI patients combat antipsychotic-induced weight gain.

\section{Acknowledgements}

This study was supported by National Institutes of Health R01 award (MH080048-02) to Dr. Cenk Tek, as well as partial support from the State of Connecticut, Department of Mental Health, and Addiction services. The authors thank Carlos Grilo, Ph.D. for help with the study design.

\section{Disclosure Statement}

The authors declare no conflicts of interest.

\section{References}

- 1 Tiihonen J, Lönnqvist J, Wahlbeck K, Klaukka T, Niskanen L, Tanskanen A, Haukka J: 11-year follow-up of mortality in patients with schizophrenia: a population-based cohort study (FIN11 study). Lancet 2009; 374:620-627.

2 Holt RIG, Peveler RC: Obesity, serious mental illness and antipsychotic drugs. Diabetes Obes Metab 2009; 11:665-679.

- 3 Sukdeb M: High prevalence of type II diabetes in schizophrenic patients. Schizophr Res 1995;15:195.

- 4 Hennekens CH, Hennekens AR, Hollar D, Casey DE: Schizophrenia and increased risks of cardiovascular disease. Am Heart J 2005;150:1115-1121.

- 5 Simon V, Van Winkel R, De Hert M: Are weight gain and metabolic side effects of atypical antipsychotics dose dependent? A literature review. J Clin Psychiatry 2009;70:1041-1050.

- 6 Brown S, Birtwistle J, Roe L, Thompson C: The unhealthy lifestyle of people with schizophrenia. Psychol Med 1999;29:697-701.

7 Wadden TA, Butryn ML, Wilson C: Lifestyle modification for the management of obesity. Gastroenterology 2007;132:2226-2238.

8 Ball MP, Coons VB, Buchanan RW: A program for treating olanzapine-RE<LATED weight GAIN. Psychiatr Serv 2001;52:967-969.

9 Littrell KH, Hilligoss NM, Kirshner CD, Petty RG, Johnson CG: The Effects of an educational intervention on antipsychotic-induced weight gain. J Nurs Scholarship 2003;35:237-241.

$>10$ Menza M, Vreeland B, Minsky S, Gara M, Radler DR, Sakowitz M: Managing atypical antipsychotic-associated weight gain: 12-month data on a multimodal weight control program. J Clin Psychiatry 2004;65: 471-477. 
-11 Vreeland B, Minsky S, Menza M, Rigassio Radler D, Roemheld-Hamm B, Stern R: A program for managing weight gain associated with atypical antipsychotics. Psychiatr Serv 2003;54:1155-1157.

-12 Jean-Baptiste M, Tek C, Liskov E, Chakunta UR, Nicholls S, Hassan AQ, Brownell KD, Wexler BE: A pilot study of a weight management program with food provision in schizophrenia. Schizophr Res 2007;96:198-205.

13 Prendergast M, Podus D, Finney J, Greenwell L, Roll J: Contingency management for treatment of substance use disorders: a meta-analysis. Addiction 2006;101:1546-1560.

14 Higgins ST, Budney AJ, Bickel WK, Foerg FE, Donham R, Badger GJ: Incentives Improve outcome in outpatient behavioral treatment of cocaine dependence. Arch Gen Psychiatry 1994;51:568-576.

15 Petry NM, Martin B, Cooney JL, Kranzler HR: Give them prizes, and they will come: Contingency management for treatment of alcohol dependence. J Consult Clin Psychol 2000;68:250-257.

-16 Sigmon SC, Steingard S, Badger GJ, Anthony SL, Higgins ST: Contingent reinforcement of marijuana abstinence among individuals with serious mental illness: a feasibility study. Exp Clin Psychopharmacol 2000; 8:509-517.

17 Petry NM, Petrakis I, Trevisan L, Wiredu G, Boutros NN, Martin B, Kosten TR: Contingency management interventions: from research to practice. Am J Psychiatry 2001;158:694-702.

18 Neeleman J, Mikhail WI: A case-control study of GP and patient-related variables associated with nonattendance at new psychiatric out-patient appointments. J Ment Health 1997;6:301-306.

19 Kidorf M, Stitzer ML, Brooner RK, Goldberg J: Contingent methadone take-home doses reinforce adjunct therapy attendance of methadone maintenance patients. Drug Alcohol Depend 1994;36:221-226.

20 Jeffery RW, Thorson C, Wing RR, Burton LR: Use of personal trainers and financial incentives to increase exercise in a behavioral weight-loss program. J Consult Clin Psychol 1998;66:777-783.

21 Mahoney MJ: Self reward and self monitoring techniques for weight control. Behav Ther 1974;5:48-57.

22 Volpp KG, John LK, Troxel AB, Norton L, Fassbender J, Loewenstein G: Financial incentive-based approaches for weight loss. JAMA 2008;300:2631-2637.

23 John LK, Loewenstein G, Troxel AB, Norton L, Fassbender JE, Volpp KG: Financial incentives for extended weight loss: a randomized, controlled trial. J Gen Intern Med 2011;26:621-626.

24 Jeffery W: Financial incentives and weight control. Prev Med 2012: doi:10.1016/j.ypmed.2011.12.024.

25 Petry NM: Contingency management: what it is and why psychiatrists should want to use it. Psychiatrist 2011;35:161-163.

26 Tidey JW: Using incentives to reduce substance use and other health risk behaviors among people with serious mental illness. Prev Med 2012: doi:10.1016/j.ypmed.2011.11.010.

27 Kohlmeier L. Dietary methodology considerations for NHANES III. Vital Health Stat 4 1992;(27):81-84.

28 Godin G, Shephard RJ: A simple method to assess exercise behavior in the community. Can J Appl Sport Sci 1985;10:141-146.

29 Woods SW: Chlorpromazine equivalent doses for the newer atypical antipsychotics. J Clin Psychiatry 2003; 64:663-667.

30 Tek C, Ratliff JC, Chwastiak L: Pharmacological treatment of obesity. Psychiatr Ann 2011;41:489-495.

-31 Paul-Ebhohimhen V, Avenell A: Systematic review of the use of financial incentives in treatments for obesity and overweight. Obes Rev 2008;9:355-367.

-32 Nestle M, Wing R, Birch L, DiSogra L, Drewnowski A, Middleton S, Sigman-Grant M, Sobal J, Winston M, Economos C: Behavioral and social influences on food choice. Nutr Rev 1998;56:S50-S74.

-33 Sooman A, Macintyre S, Anderson A: Scotland's health - a more difficult challenge for some? The price and availability of healthy foods in socially contrasting localities in the west of Scotland. Health Bull 1993;51: 276-284.

-34 Wing RR, Epstein LH, Marcus M, Shapira B: Strong monetary contingencies for weight loss during treatment and maintenance. Behav Therapy 1981;12:702-710.

35 John LK, Loewenstein G, Volpp KG: Empirical observations on long-term use of incentives for weight loss. Prev Med 2012: doi:10.1016/j.ypmed.2012.01.022.

36 Kahneman D, Tversky A: Choices, values, and frames. Am Psychol 1984;39:341-350.

37 Wadden TA, Butryn ML, Byrne KJ: Efficacy of lifestyle modification for long-term weight control. Obes Res 2004;12(suppl):151S-62S.

38 Ganguli R, Strassnig M: Prevention of metabolic syndrome in serious mental illness. Psychiatr Clin North Am 2011;34:109-125.

-39 Wu M-K, Wang C-K, Bai Y-M, Huang C-Y, Lee S-D: Outcomes of obese, clozapine-treated inpatients with schizophrenia placed on a six-month diet and physical activity program. Psychiatr Serv 2007;58:544-550.

40 Petry NM: Contingency management treatments: controversies and challenges. Addiction 2010;105:15071509. 